\title{
Design for Manufacturability (DFM) of 3D Printed Parts Fabricated Using Open Source 3D Printer
}

\author{
N.A. Maidin ${ }^{1 *}$, M.H.A. Rahman ${ }^{1}$, M.N. Ahmad ${ }^{1}$, M.B.M. Salahuddin ${ }^{3}$, S.N.H \\ Mazlan $^{2}$, M.K. Wahid ${ }^{1}$, M.H. Osman ${ }^{1}$ and R. Jumaidin ${ }^{1}$
}

${ }^{1}$ Faculty of Engineering Technology,

Universiti Teknikal Malaysia Melaka, Hang Tuah Jaya, 76100 Durian Tunggal, Melaka, MALAYSIA

${ }^{2}$ Faculty of Mechanical Engineering,

Universiti Teknikal Malaysia Melaka, Hang Tuah Jaya, 76100 Durian Tunggal, Melaka, MALAYSIA

${ }^{3}$ Faculty of Engineering, Universiti Putra Malaysia, 43400 Serdang, Selangor, MALAYSIA

*Corresponding email

DOI: https://doi.org/10.30880/ijie.2020.12.05.025

Received 3 February 2020; Accepted 26 June 2020; Available online 30 June 2020

\begin{abstract}
Fused deposition modeling (FDM) is one of the well-known additive manufacturing (AM) techniques to fabricate the part using layer-by-layer concept. Recently, an open source 3D printer is become widely available used by 3D printer user because of its affordability and portability. In this study, the performance of an open source 3D printer was evaluated based on the dimensional accuracy of the printed parts. The test model was fabricated using two types of printer, which is low cost 3D printer, Prusa and mid-end 3D printer, Cubepro. Then, the dimension of every model structure was measured using Rexscan 3D laser scanner and was compared.
\end{abstract}

Keywords: Fused Deposition Modeling (FDM); 3D Laser Scanner and Dimensional Accuracy

\section{Introduction}

Additive manufacturing (AM) is described as the technology that fabricated the parts layer-by layer by using a material such as plastic, metal or concrete. Commonly, using this process, a 3D CAD modeling was converted into STL.file and was sliced to certain parameter setting before the printing process occurs. AM technologies started in 1980 's to fabricate the parts in precise geometry with shortened manufacturing time. In AM, there are a few printing techniques available such as stereolithography (SLA), selective laser sintering (SLS), laminated object manufacturing (LOM) and fused deposition modeling (FDM). However, the most commonly used technique among AM is FDM because the technology is cheaper compared to others [1]. However, the drawback of this technique it is inferior in terms of printed part's quality including tensile strength, surface finishing and dimensional accuracy. For certain crucial prototypes, despite tensile strength, part's accuracy are also important factors to consider. Various AM benchmarks parts have been designed and developed in the last decade, purposely to evaluate the performance of specific AM process [2-3]. The objective to develop the benchmark parts and benchmarking procedure is to evaluate the performance of FDM systems in terms of geometric features for specific functional requirement.

Several benchmark studies have been carried out to determine the levels of dimensional accuracy and also surface quality by measuring the surface roughness that is achievable with the current AM process [4-5]. Various test parts have been designed for the benchmark study and most of its typically involve the fabrication of one sample that have several design processes [6]. The benchmarking evaluation starts to revolve in 1991 by Kruth [7] and the test parts that 
have an inverted U- frame possessed several geometric features such as cylindrical shell, inclined cylinders and pegs and overhangs. The test parts have its largest dimension by only $100 \mathrm{~mm}$ as compared to the others build size available in most of the machines. The benchmark part with rich in fine and medium size features was also purposed as the features contain of the recessed fins and cantilevers, but this part is difficult to test on the coordinate measuring machine (CMM) for the dimensional accuracy test [8]. The benchmark is also used to examine both linear accuracy and feature repeatability of AM parts with four others AM technique. The benchmark consists on repeated features and dimension varying scales for evaluating respective to the accuracy of features in different dimensional scales [9]. The comparative evaluation of AM systems and processes was demonstrated to evaluate the performance of four-well known RP process which is SLA, SLS, FDM and LOM and it was fabricated by default machine parameters and settings. The features contain flatness and straightness, overhang, roundness and also concentricity, and most of the features are designed to test on the accuracy and linear accuracy [10].

Injection molding process manufacturing has been known as the excellent way to bring out the best of plastic manufacturing process. It was patented by John W.Hyatt in 1872 and since then, the process was established and well known in fabrication of thermoplastic material. Three areas that must be notified to ensure the successful application of plastic parts are designed, product and process. These three teams are interrelated and appropriate rules when designing and fabricating a part. The designers also need to ensure that the design and process is capable of meeting the design requirement such as size, shape, details and tolerances. In 2013, Adam and Zimmer, had studied on "Direct Manufacturing Design Rules" which provided the user of 3D printing and FDM.

Based on their study, the guide for the design and their limitation are presented. The researchers segregated the design into 3 different categories which are the basic element, aggregated element and transition element. In AM, part was fabricated by adding material layer by layer, hence parts that does not have the base faced difficulties in its processing. Thus, Design for Manufacturing (DFM) was introduced to evaluate how well that the design can be manufactured with a good functionality [12]. The first phase in DFM is designing a concept design [13], thus in AM, the impact of DFM decisions would result in the mechanical properties and the abilities of the machine to produce the product because it will effect on the product development [14]. Therefore, in this research the manufacturability of widely used open source 3D printer is explored.

\section{Research Methodology}

\subsection{CAD Development}

The geometrical representation of test model was generated by SolidWorks. The CAD data were then converted into STL format. The fabrication for the build of the model and its support structure was generated automatically. The selection of the design shapes for the test model had been discussed from the previous researchers study. The features were also being varied by different size. In this study, two type of $3 \mathrm{D}$ printer was being evaluated in terms of manufacturability which is, UP Plus2 (low cost 3D printer) and CubePro 3D printer (mid-end 3D printer). The benchmarking model and feature description was presented in Fig. 1.

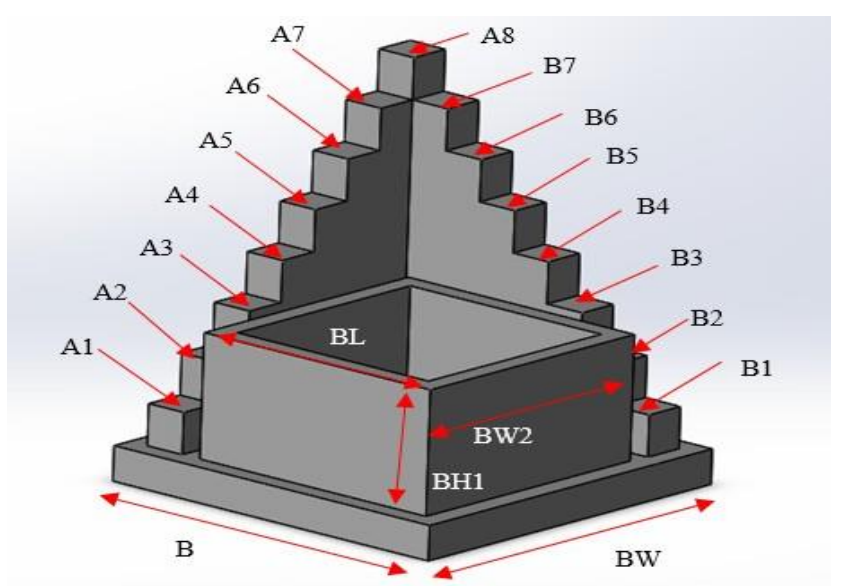

Fig. 1 - CAD Visual Presentation for Benchmarking Model

\subsection{Model Development using Open Source 3D printer}

The benchmarking model was fabricated by Up Plus2 3D printer and CubePro 3D printer. The sample building time is $4 \mathrm{hr} 49$ minute for Up Plus2 3D printer, meanwhile it takes $2 \mathrm{hr}$ using CubePro 3D printer. The fabricated part was shown in Fig. 2. Both of the parts were successfully fabricated using both type of 3D printers. Later on, the dimensional accuracy of the parts was determined by using laser scanning and analyzed through inspection software, Geomagic. The tolerance set for each 3D printer was $\pm 1.000 \mathrm{~mm}$ 
Table 1 - Geometric features benchmarking model

\begin{tabular}{|c|c|c|c|}
\hline Feature & ID & Descriptions & Evaluation \\
\hline Base & $\mathrm{BL}, \mathrm{BW}$ & $90 \times 90 \mathrm{~mm}$ & $\begin{array}{l}\text { Flatness, } \mathrm{X} \text { and } \mathrm{Y} \text { line } \\
\text { accuracy }\end{array}$ \\
\hline Stairs / Steps & $\begin{array}{l}\mathrm{A} 1-\mathrm{A} 8 \\
\mathrm{~B} 1-\mathrm{B} 7\end{array}$ & $\begin{array}{l}\text { Steps are connected to from a } \\
\text { stair and their height for each of } \\
\text { the stairs is } 11.8 \mathrm{~m} \text {. Base for } \\
\text { each of the stairs is } 10 \times 10 \mathrm{~mm} \text {. }\end{array}$ & $\begin{array}{l}\text { Staircase effects, rectangular } \\
\text { blocks, extruded bosses. }\end{array}$ \\
\hline \multirow{3}{*}{ Rectangular boss } & BLU & $64.48 \mathrm{~mm}$ & \multirow{3}{*}{$\begin{array}{l}\text { Flatness. X and Y line } \\
\text { accuracy }\end{array}$} \\
\hline & BW2 & $65 \mathrm{~mm}$ & \\
\hline & BH1 & 35.625 & \\
\hline
\end{tabular}

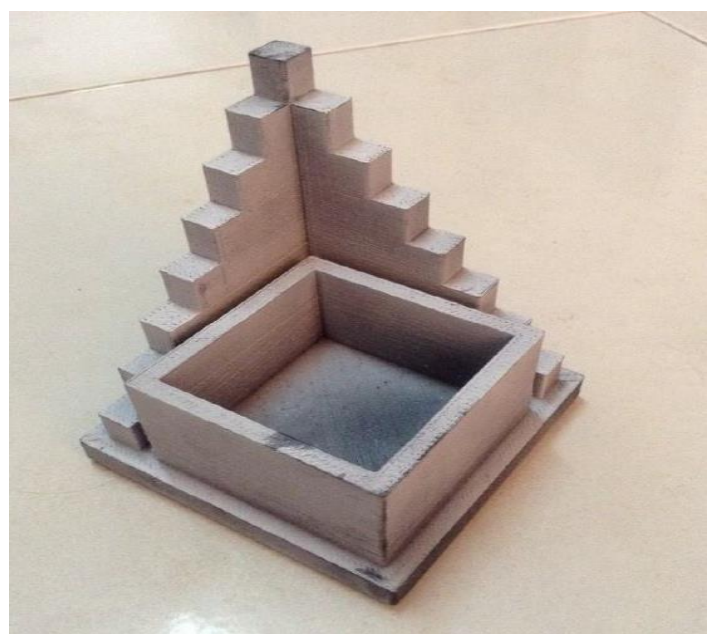

(a)

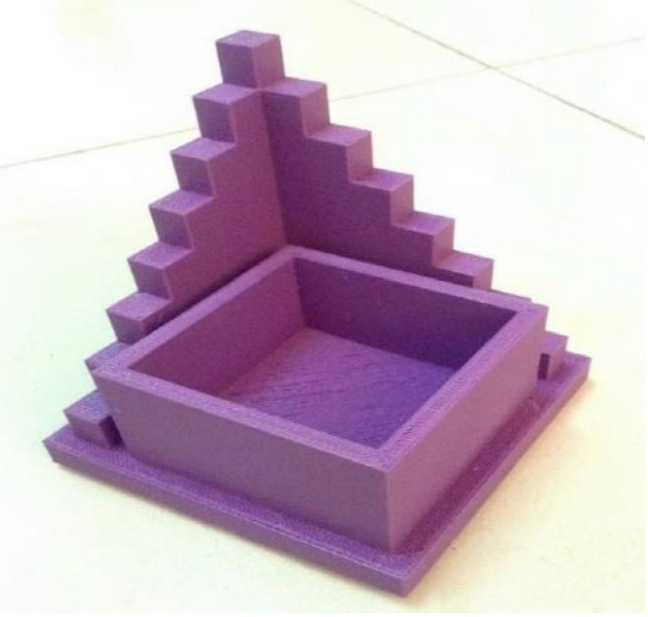

(b)

Fig. 2 - (a) Fabricated part by using Up Plus2 3D printer; (b) Fabricated by CubePro 3D printer

\subsection{D Laser Scanning}

A laser scanning approach is suitable for analyzing the parts created utilizing AM. Generally, 3D laser filtering frameworks are relatively less accurate than CMM. In this study, the CMM machine was not used because accessibility limitation of CMM probe at certain part. Nevertheless, the laser scanning had a precision of $0.0749 \mathrm{~mm}$ which is acceptable for this study.

\section{Results and Discussion}

\subsection{Evaluation of benchmarking model using UP Plus 3D Printer}

Fig.3, presents the comparison between the CAD data and printed part by using UP Plus 3D printer. Some of observation from the fabricated model are:

a) Warping along the edges of the box on the base feature.

b) The part was successfully fabricated, but the existence of gap between box and stair wall too close that make the laser scanning cannot detect the complex geometry.

Based on scanning of the benchmark part model, 2,654,299 points were collected. Fig.4 (a-d) shows that the subsequent error (deviation) maps for various orientations of the benchmarking model. Thus, the areas of the model in green are within $+/-0.0250 \mathrm{~mm}$ of the CAD model, red areas are outside the CAD model (oversized) by $0.5000 \mathrm{~mm}$ or greater, and areas in blue are inside the CAD model (undersized) by $-0.5000 \mathrm{~mm}$ or less. The maximum deviation is $+/-$ $0.4999 /-0.5000 \mathrm{~mm}$, with average $+/-0.1284 /-0.1568 \mathrm{~mm}$, the standard deviation for the data is $0.1645 \mathrm{~mm}$ standard deviations and RMS estimate is $0.1735 \mathrm{~mm}$. Comparison of benchmark model has been performed using Geo-Magic Qualify Software and the error maps is shown in Fig. 4. 


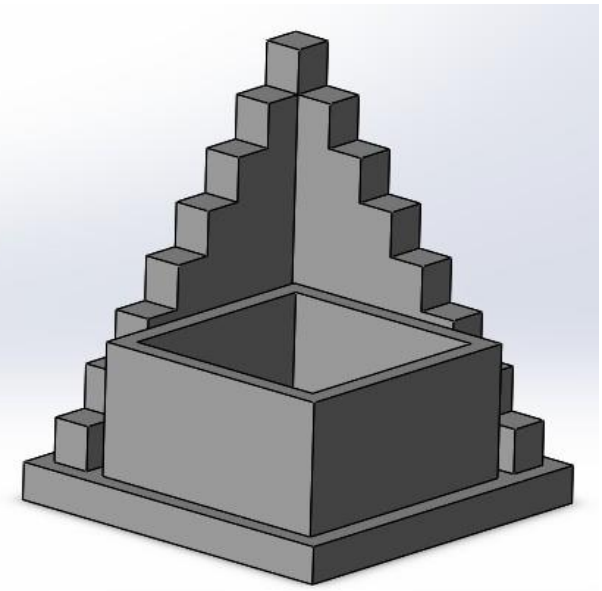

(a)

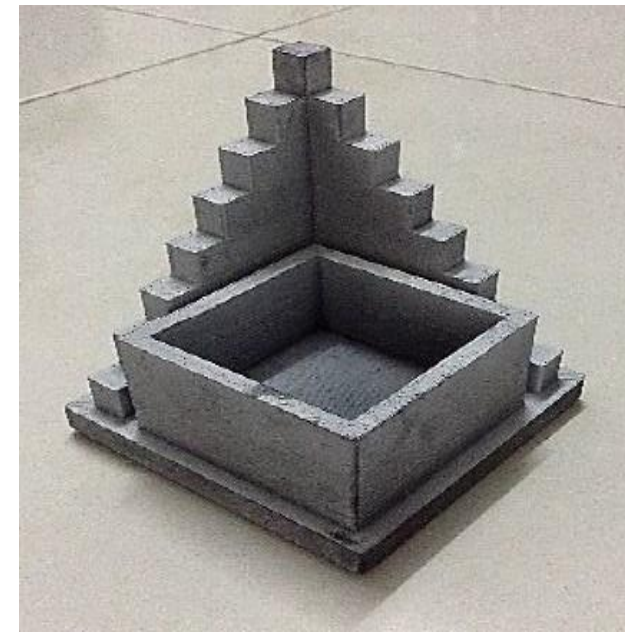

(b)

Fig. 3 - Fabricated model using UP Plus2 3D printer

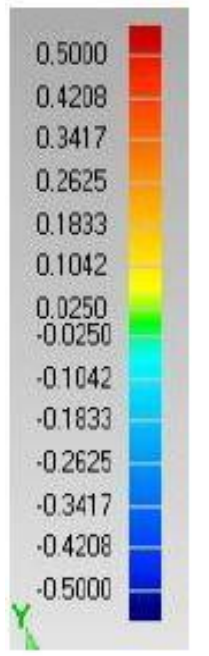

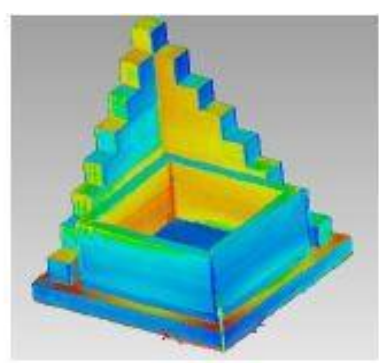

a)

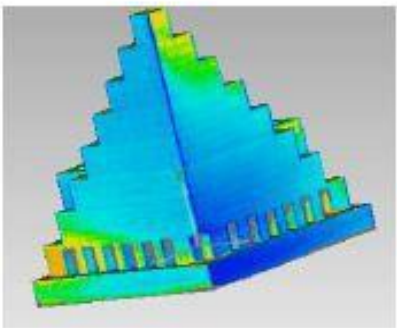

c)

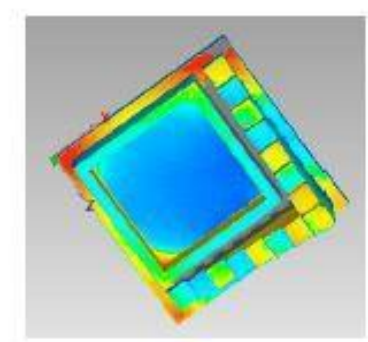

b)

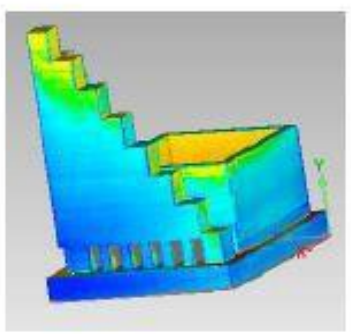

d)

Fig. 4 - 3D Comparisons of fabricated benchmark model printed by using UP Plus 3D printer to CAD dimension (unit: mm)

\subsection{Evaluation of benchmark model fabricated using Cube Pro 3D Printer}

Fig. 5 (a) presents the original CAD model and fabricated benchmark model (b-d). Below are observations based on visual examination of the benchmark part model:

a) Benchmarking successful fabricated, but warping along the edges of the bottom of the base feature.

b) The part was successfully fabricated, but the gap between box and stair wall too close that make laser scanning cannot detect the complex geometry.

c) Warping occurred especially at overhangs parts.

A sum of 2,551,369 point was collected from benchmarking part model. Figure 6 (a-d) indicates that the subsequent error (deviation) maps for various orientations of the benchmarking model. Thus, the areas of the model in green are within $+/-0.0250 \mathrm{~mm}$ of the CAD model, red areas are outside the CAD model (oversized) by $0.5000 \mathrm{~mm}$ or greater, and areas in blue are inside the CAD model (undersized) by $-0.5000 \mathrm{~mm}$ or less. The maximum deviation is $+/-$ $0.4999 /-0.5000 \mathrm{~mm}$, with average $+/-0.1654 /-0.2161 \mathrm{~mm}$, the standard deviation for the data is $0.2173 \mathrm{~mm}$ standard deviations and RMS estimate is $0.2390 \mathrm{~mm}$. Observation was made and further inspection by using Geo-Magic Qualify 
Software and its error maps is indicating in Fig. 6.

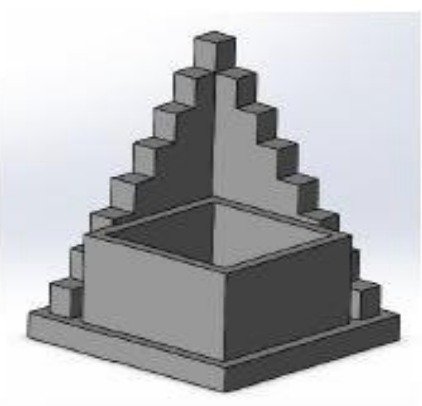

a)

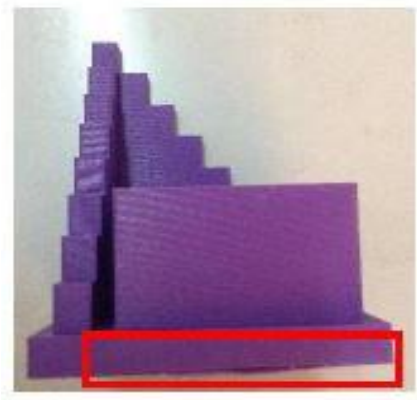

c)

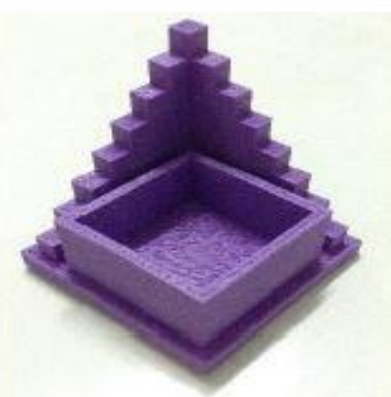

b)

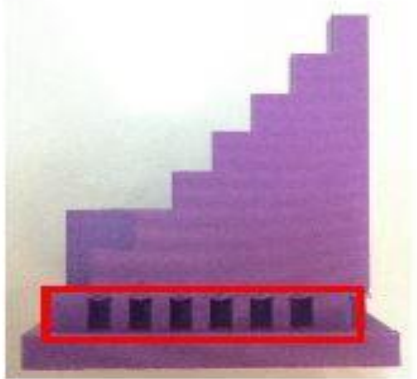

d)

Fig. 5 - View of benchmarking fabricated model in 3D CAD and fabricated part versions of benchmarking model
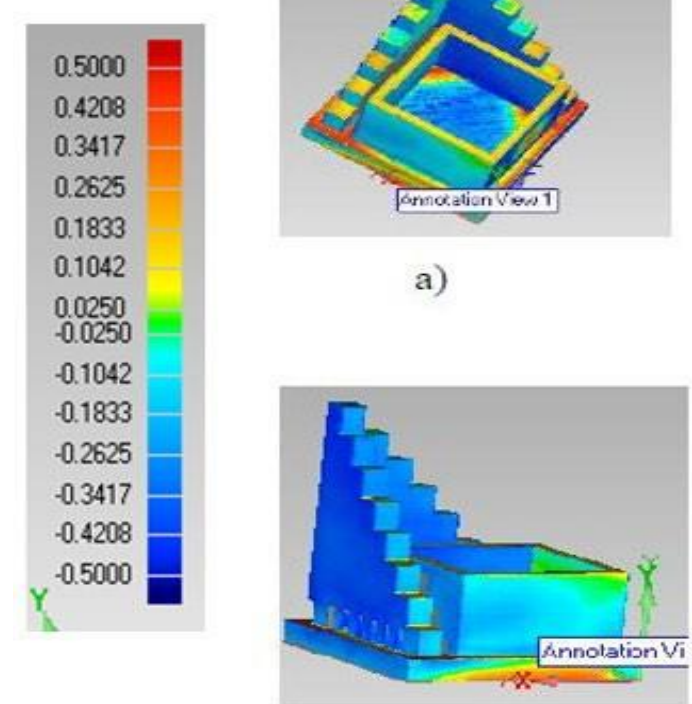

c)

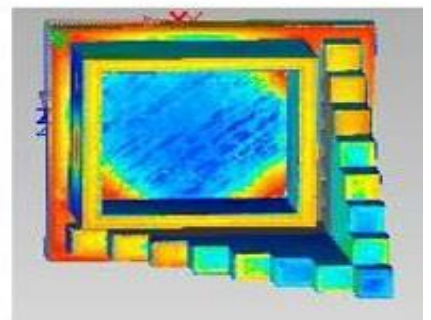

b)

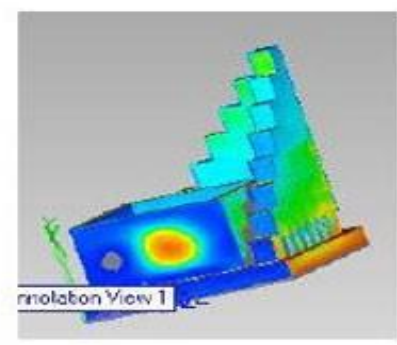

d)

Fig. 6 - 3D Comparisons of fabricated benchmark model printed by using CubePro 3D printer to CAD dimension (unit: mm)

Based on Figure 7, the comparison between two types of 3D printer machine is shown. Features BW, A2, A3, A5, A6, A8, B5, B7, BW2 and BH1 shows that the dimension of benchmarking part fabricated by CubePro 3D printer machine are undersized from $\mathrm{CAD}$ data. While, features $\mathrm{BL}, \mathrm{B} 3$ and $\mathrm{BLU}$ produced oversized printed part from $\mathrm{CAD}$ data. Then, for features A1, A4, A7, B1, B2, B4 and B6 printed by CubePro shows that the dimension is close to CAD data 
Thus, other than that almost all dimensions are close to CAD data printed by UP Plus2 3D printer. This means that UP Plus2 3D printer are capable to print more accurate than CubePro 3D printer machine.

\section{Conclusion}

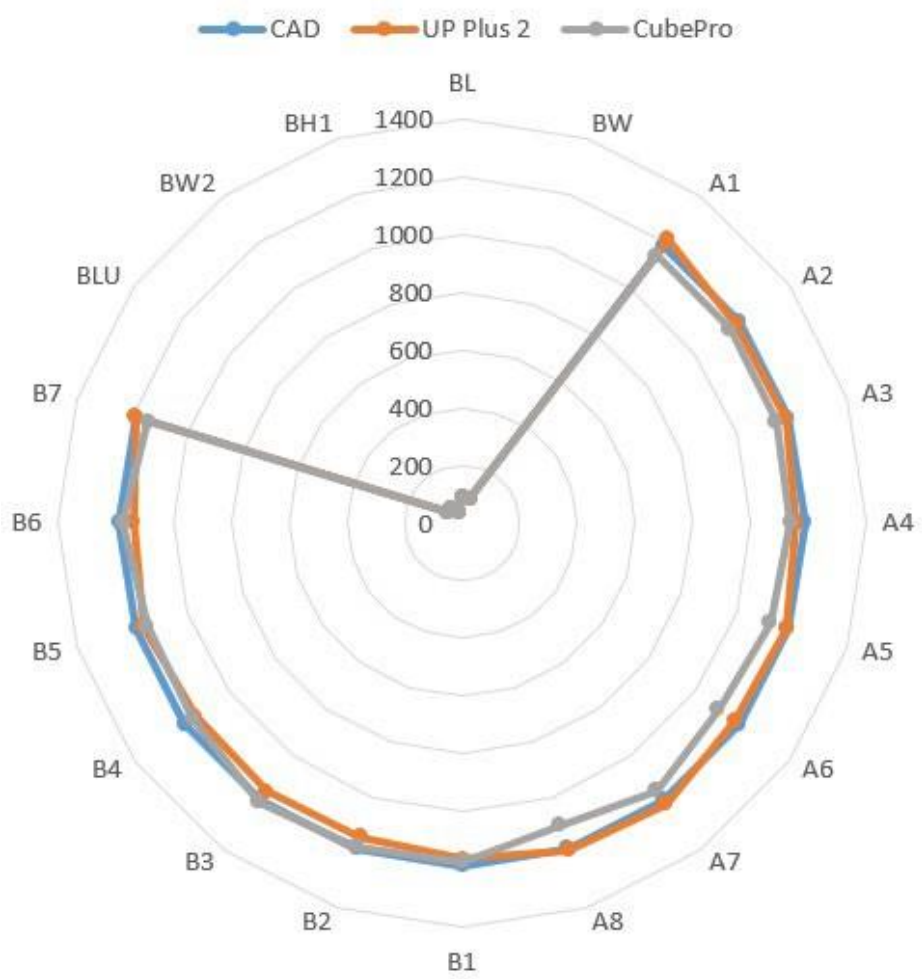

Fig. 7 - Comparison of deviation from CAD for different types of 3D printers

Based on the result obtained, it shows that by using an entry level 3D printer can produce relatively good and accurate dimension part model. Dimension accuracy is an important criteria to produce a good and quality product. Good design if this is fulfilled, it means customer's need and customer satisfactions improved.

\section{Acknowledgement}

The authors wish to thank Universiti Teknikal Malaysia Melaka (UTeM) and Ministry of Higher Education Malaysia for supporting this research (RAGS/1/2015/TK0/FTK/03/B00113).

\section{References}

[1] A. M. Technology, "Fused Deposition Modeling," Castle Island's Worldwide Guide to Rapid Prototyping, vol. 220, no. v, pp. 2-4, 2012.

[2] "A novel methodology of design for Additive Manufacturing applied to Additive Laser Manufacturing Process." 2014.

[3] Abdul Haq, R. H., Marwah, O. M. F., Abdol Rahman, M. N., Ho, F. H., Abdullah, H., Ahmad, S., Tajul Arifin, A. M., Hassan, M. F., \& Yunos, M. Z. (2018). Mechanical Properties of PCL/PLA/PEG composite blended with different molecular weight (MW) of PEG for Fused Deposition Modelling (FDM) filament wire. International Journal of Integrated Engineering, 10(5).

[4] P. Jain and A. M. Kuthe, "Feasibility study of manufacturing using rapid prototyping: FDM approach," Procedia Engineering., vol. 63, pp. 4-11, 2013.

[5] Johar, M., Wong, K. J., \& Tamin, M. N. (2018). Non-Fickian Absorption Characteristics of Adhesive Joints: Capillary Effects and Residual Properties. International Journal of Integrated Engineering, 10(5).

[6] T. Gornet, "History of additive manufacturing Introduction of non-SL systems Introduction of low-cost 3D printers," pp. 1-34, 2014.

[7] K. Papers, "Material Incress Manufacturing by Rapid Prototyping Techniques," vol. 40, 1991.

[8] D. Pham and R. Gault, “A comparison of rapid prototyping technologies,” International Journal Machine Tools Manufacturing, vol. 38, no. 10-11, pp. 1257-1287, 1998.

[9] T. H. C. Childs and N. P. Juster, "Linear and Geometric Accuracies from Layer Manufacturing," CIRP Ann. Manufing Technology, vol. 43, no. 1, pp. 163-166, 1994. 
[10] M. Mahesh, Y. S. Wong, J. Y. H. Fuh, and H. T. Loh, "Benchmarking for comparative evaluation of RP systems and processes," Rapid Prototyping Journal, vol. 10, no. 2, pp. 123-135, 2004.

[11] G. A. O. Adam and D. Zimmer, "Design for Additive Manufacturing-Element transitions and aggregated structures," CIRP Journal Manufacturing Science Technology, vol. 7, no. 1, pp. 20-28, 2014.

[12] P. Jain and A. M. Kuthe, "Feasibility study of manufacturing using rapid prototyping: FDM approach," Procedia Engineering, vol. 63, pp. 4-11, 2013.

[13] C. C. Kuo and S. J. Su, "A simple method for improving surface quality of rapid prototype," Indian Journal Engineering Material Science, vol. 20, no. 6, pp. 465-470, 2013.

[14] Maidin, N.A.B., ab Rahman, M.H.B., Ahmad, M.N.B., Osman, M.H.B., Wahid, M.K.B., Hussin, M.S.F.B., Yassim, H.M.B., Razali, M.Z.A.B., (2018). A Prototype Development of Anti-Hunchback Device. Journal of Mechanical Engineering, Vol SI (1), 192-209. 